\title{
Estimating Age at Death Based on Costal Cartilage Calcification
}

\author{
Tomoya Ikeda ${ }^{1,2}$ \\ ${ }^{1}$ Department of Forensic Medicine, Tohoku University Graduate School of Medicine, Sendai, Miyagi, Japan \\ ${ }^{2}$ Department of Legal Medicine, Osaka City University Medical School, Osaka, Osaka, Japan
}

\begin{abstract}
Age estimation is a crucial part of forensic investigations. Because different parts of the body are often found at crime scenes, it is important to explore the regions that can be used for age estimation. Previous studies have used simple X-ray to analyze changes in costal cartilage calcification as a measure of age. Here, we tested whether age could be better estimated using measurements of costal cartilage calcification on postmortem CT images. In this study, male and female decedents ( $n=10$ each) from autopsy cases were grouped into 10-year incremental age groups (20-29 y; 30-39 y; up to $89 \mathrm{y})$. We found that the mean Hounsfield unit (CT number) and percentage calcification (the ratio of the ossified area to the whole area) increased with age for both sexes. However, there were marked individual differences within many of the groups, and this led to a statistically significant difference $(P<0.05)$ only between the $20-29$ y group and the older age groups. To improve the ability to correctly assign cases to age groups, we introduced and reanalyzed the data using Bayesian statistics. This improved the classifications rates, with $40 \%$ of males and $35 \%$ of females correctly assigned into their age groups. Broadening the age range could further improve the number of matches. Thus, combining Bayesian statistics with CT imaging can be used to estimate age at death from costal cartilage calcification, and could be used as an adjunct in forensic investigations.
\end{abstract}

Keywords: age estimation; Bayesian statistics; calcification; computed tomography; costal cartilage Tohoku J. Exp. Med., 2017 December, 243 (4), 237-246. C 2017 Tohoku University Medical Press

\section{Introduction}

Previous morphological studies have investigated estimations of age and sex from cadaveric ribs to establish baseline values for the identification of unidentified decedents. Iscan et al. (1984a) found that the sternal end of the fourth rib undergoes age-related metamorphosis. They classified this process into nine stages for each sex (Iscan et al. 1984b, 1985). Similarly, Kunos and colleagues (1999) sought to identify metrical and morphological characteristics of the costal face, tubercle, and head of the first rib. $\mathrm{X}$-ray photograms make it possible to examine the entire costal cartilage for age- and sex-related differences in calcification, and images can be collected from both living and dead persons (Stewart and McCormick 1984; Rejtarova et al. 2004; Garamendi et al. 2011). However, morphological classification with the naked eye or on an X-ray requires advanced skills and experience. Over the past few years, the use of computed tomography (CT) has increased in many forensic institutions in Japan, and advances in software have made the creation of three-dimensional (3D) images relatively easy. These images can be stored digitally and used as a source of objective data, for example, the Hounsfield unit (HU). The HU scale is a quantitative, standardized scale that describes radiodensity as CT numbers. These 3D images can be used to extract target areas by deleting overlapping structures that can disrupt the analysis of a plain radiograph. In this study, we explored a new methodology for age estimation of costal cartilage calcification using $\mathrm{HU}$ and the volume ratio of classification areas. Following this, we used a Bayesian theorem to "narrow down" the age estimation range.

In this study, we chose to examine the second and fourth costal cartilages in adults. McCormick (1980) investigated X-ray photographs of costal cartilages from 210 bodies and found that mineralization of the costal cartilages initially appeared at 15 years but was uncommon before the age of 20 years, and show that marked calcification appears after 50 years of age. Calcification can be observed histologically during the first decade of life, and radiologically after the second decade (Rejtarova et al. 2009). Many studies have used the fourth costal cartilage for estimating age (Iscan et al. 1984a, b, 1985; Inoi 1997; Dedouit et al. 2008; Fanton et al. 2010; Verzeletti et al. 2013; Cerezo-Roman and Hernandez Espinoza 2014; Haj Salem et al. 2014), and some researchers have analyzed the first ribs (King 1939; Kampen et al. 1995; Barchilon et al. 1996; Kunos et al. 1999), showing that calcification of the costal cartilage of

Received July 31, 2017; revised and accepted November 13, 2017. Published online December 5, 2017; doi: 10.1620/tjem.243.237.

Correspondence: Tomoya Ikeda, Department of Legal Medicine, Osaka City University Medical School, 1-4-3 Asahi-machi, Abeno-ku,

Osaka, Osaka 545-8585, Japan.

e-mail: ikeda.tomoya@med.osaka-cu.ac.jp 
the first rib is different to that of the other ribs (King 1939; Kampen et al. 1995; Barchilon et al. 1996; Kunos et al. 1999). However, the costal cartilage of the first rib overlaps with the clavicle and soft tissue, and this makes it difficult to extract only the first costal cartilage from a CT image. Below the fourth costal cartilage, there is often fusion with the subsequent costal cartilage, so the boundary between the two areas of cartilage is too ambiguous.

Bayesian estimation was first reported by Lucy et al. (1996) using teeth as a way to overcome constraints with using regression analyses. Here, we report for the first time a method for combining postmortem CT data and Bayesian statistics in evaluating calcification of costal cartilage with aging.

\section{Materials and Methods}

Study cases

In this study, 10 decedents of each sex in each age group (10year increments from 20 s to 80 s) were chosen from among autopsy cases, for which postmortem CT examination had been performed at our institution from January 2013 to September 2015. However, we only had six females in their 20 s, because there were fewer cases in this age bracket during our research period. In total, we analyzed radiographic images from 136 individuals ( 70 males and 66 females).

To determine inter-observer error, 10 cases were chosen from autopsy cases at our institution from November 2015 to May 2016. To assess our age estimation table using Bayesian statistics, an additional 20 males and 20 females were chosen from our autopsy cases at our institution from November 2015 to May 2016, with 1 to 4 individuals in each age group.

To exclude putrefactive artifacts, we analyzed scans performed no later than 3 weeks after death. In addition, as a general rule, severely decomposed bodies do not undergo CT scanning at our institution. Cases with chest injuries, including charred bodies, were also excluded from this study. The study was approved by the Ethics Committee of Tohoku University Graduate School of Medicine (2015-1-410).

\section{CT protocol and image management}

A 64-channel (i.e., 64 detector rows with a section thickness of $0.5 \mathrm{~mm}$ at the center of rotation) Aquilion Multi Detector (MD) CT scanner (Toshiba Medical Systems, Tokyo, Japan) was used for imaging in this study. Noncontrast volumetric helical scans were acquired from the vertex of the head to the pelvis at $120 \mathrm{kVp}$ or $135 \mathrm{kVp}$ with variable mAs. We used an auto-exposure control system, a beam pitch of 0.844 , a scanning field-of-view diameter of $500 \mathrm{~mm}$, and a section thickness of $1.0 \mathrm{~mm}$ in 32-slice mode. The MDCT datasets were sent to a server for digital imaging and communication in medicine (POP-Net Server; Image ONE, Tokyo, Japan). Multi-planar reconstruction images, volume rendering (VR) images, and maximum intensity projection (MIP) images were reconstructed using a $3 \mathrm{D}$ workstation (Ziostation2 ver. 2.1.5.0; Ziosoft, Tokyo, Japan).

\section{Analysis of costal cartilage in CT images}

The costal cartilages from both the second and fourth ribs were analyzed for each case. A target area of bone was chosen from the VR images (Fig. 1a). The image was changed to an MIP with a window width (WW) and a window level (WL) of 450 and 1,500, respec- tively (Fig. 1b). One costal cartilage, including the sternum and the rib, was manually extracted from the image. At this stage, an observer judged the presence or absence of calcification close to the area of sternal attachment. The presence of calcification was determined by visible areas of X-ray absorption-even for a short interval, regardless of shape. Next, to erase the sternum and the rib, we manually extracted only the costal cartilage (Fig. 1c). The image was then changed to WL 80 (Fig. 1d). The total volume of the costal cartilage and whole mean HU were automatically measured using the workstation. Finally, the WL was then changed to 180 to analyze only the area of calcification in the costal cartilage (Fig. 1e). The volume of the ossified area was automatically measured by the workstation. We calculated the percentage of calcification as the ratio of the volume of the ossified area to the volume of the entire area.

\section{Assessment of inter-observer error}

To assess inter-observer error, we chose two individuals who had not previously used the 3D workstation to analyze the second and fourth costal cartilages of both ribs from 10 randomly chosen cases that were not part of the 136 study cases. The methods were explained to these individuals both verbally and with written documentation. I also analyzed the samples. The three observers calculated the mean $\mathrm{HU}$ and percentage of calcification for each cartilage, and the numerical values were examined by Intraclass Correlation Coefficient (ICC) analysis. The presence or absence of calcification close to the sternal attachment area was also judged by these three observers.

\section{Statistical analysis}

The Mann-Whitney $U$ test and the Kruskal-Wallis test: The mean HU and percentage of calcification are not normally distributed. The Mann-Whitney $U$ test was used to determine significant sex differences for the mean $\mathrm{HU}$ and percentage of calcification for each cartilage in the 10-year groups. The Kruskal-Wallis test was used to determine significant differences in mean HU and percentage of calcification for each costal cartilage in each 10 -year group by sex. $P<$ 0.05 was considered to indicate statistical significance. Analyses were carried out with Microsoft Excel 2010 with the Analysis ToolPak add-in.

\section{Bayesian statistics}

Using Bayes' theorem, we created an estimation table showing the distribution of posterior probability in each age group. Bayesian statistics consist of prior probability, likelihood, and posterior probability. In age estimation, prior probability, $P$ (Age $i)$, is the probability of an individual belonging to a defined age group without any additional information (Lucy et al. 1996). In this study, $P$ (Agei) was the prior probability based on the age distribution of consecutive forensic autopsy cases for which postmortem CT was performed at our institution from January 2013 to September $2015(n=1,206 ; 783$ males, 424 females; Table 1). The 136 cases that were part of this study were not included in the prior probability distribution. The likelihood, $P(\mathrm{I} \mid$ Age $i)$, is the conditional probability of the observed information, I, given any hypothesis is true (Lucy et al. 1996). In this study, $P(I \mid$ Age $i)$ indicates the probability of that the proportion of the observed average HU (or ossification rate) exists within a specified average $\mathrm{HU}$ (or ossification ratio) distribution in each age group.

The posterior probability, $P($ Age $i \mid \mathrm{I})$, is the conditional probability of the hypothesis being true given the observed information (Lucy 


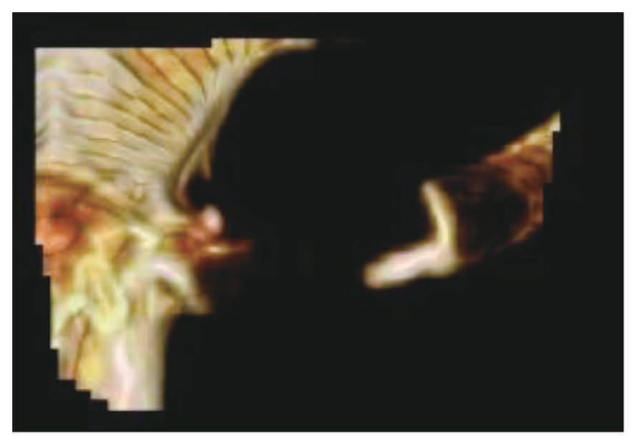

(a)

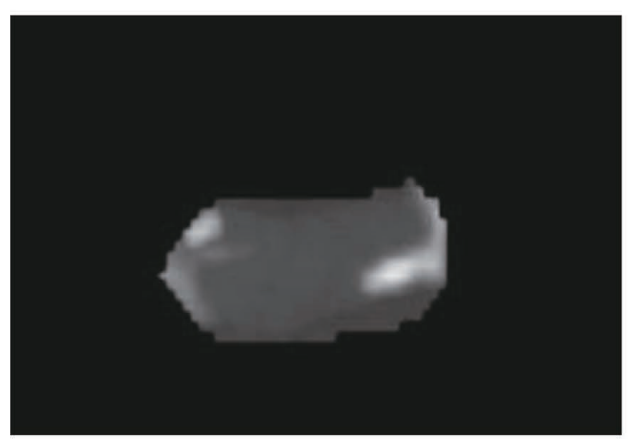

(c)

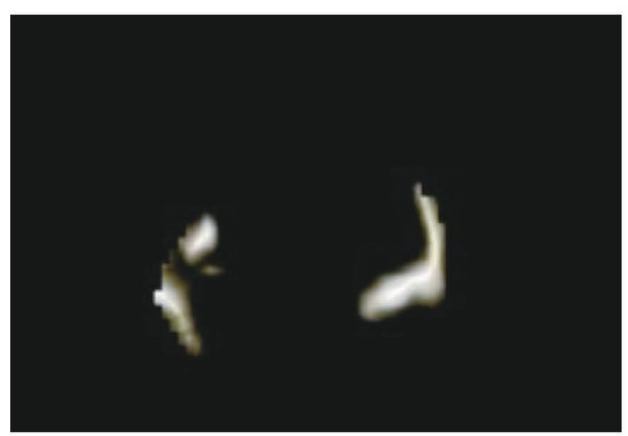

(e)

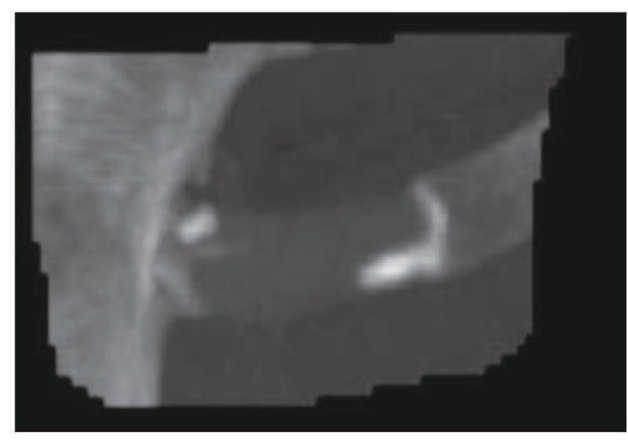

(b)

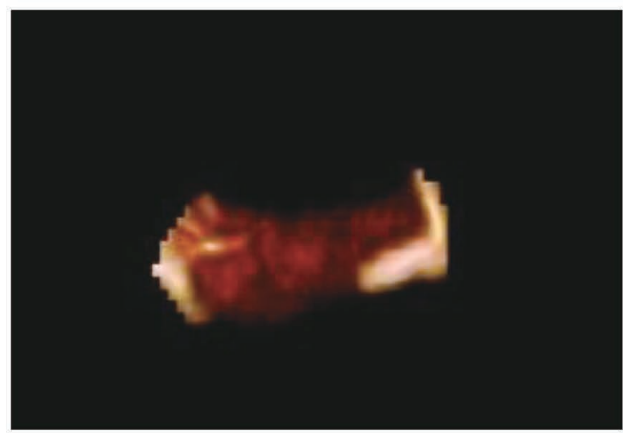

(d)

Fig. 1. Extracting the target area using the workstation.

This case was an 84-year-old female. (a) The left second costal cartilage, including the sternum and the rib, was manually extracted using volume rendering (VR). (b) Maximum intensity projection image with a window width (WW) of 450 and a window level (WL) of 1,500. (c) Manual extraction of costal cartilage from the image in (b). (d) Automatic extraction of costal cartilage from the VR image with a WL of 80. (e) Automatic extraction of the area of calcification from the VR image with a WL of 180.

et al. 1996). In other words, $P$ (Age $i \mid I)$ is the probability of the individual being in a particular age group $i$ if the mean HU $I$ is observed. We use $j$ to refer to all of the $i$ groups together (Sakaue 2015).

Bayes' theorem states that the posterior probability is proportional to the prior probability multiplied by the likelihood (Lucy et al. 1996; Sakaue 2015). The formula can be expressed as follows:

$$
P\left(A g e_{i} \mid I\right)=\frac{P\left(A g e_{i}\right) \times P\left(I \mid A g e_{i}\right)}{\sum P\left(A g e_{j}\right) \times P\left(I \mid A g e_{j}\right)}
$$

If several values are used, Equation 1 can be rewritten as:

$$
P\left(\text { Age }_{i} \mid I_{1}, I_{2}, \ldots I_{n}\right)=\frac{P\left(\text { Age }_{i}\right) \times P\left(I_{1}, I_{2}, \ldots I_{n} \mid A g e_{i}\right)}{\sum P\left(A g e_{j}\right) \times P\left(I_{1}, I_{2}, \ldots I_{n} \mid A g e_{j}\right)}
$$

By assuming conditional independence of the observed measurements in each age group, Equation (2) can be rewritten (Lucy et al. 1996; Sakaue 2015) as:

$$
P\left(\text { Age }_{i} \mid I_{1}, I_{2}, \ldots I_{n}\right)=
$$


Table 1. Prior probability using male and female populations.

\begin{tabular}{|c|c|c|c|c|c|c|c|}
\hline Age (years) & $20-29$ & $30-39$ & $40-49$ & $50-59$ & $60-69$ & $70-79$ & $80-89$ \\
\hline \hline $\begin{array}{c}\text { Autopsy } \\
\text { Male bodies } \\
(\mathrm{n}=782)\end{array}$ & $6.01 \%$ & $10.87 \%$ & $9.72 \%$ & $18.80 \%$ & $20.84 \%$ & $16.24 \%$ & $9.34 \%$ \\
\hline $\begin{array}{c}\text { Autopsy } \\
(\mathrm{n}=47)\end{array}$ & $6.84 \%$ & $7.79 \%$ & $10.85 \%$ & $11.32 \%$ & $11.79 \%$ & $15.57 \%$ & $22.17 \%$ \\
$\begin{array}{c}\text { Female bodies } \\
(\mathrm{n}=424)\end{array}$ & $(\mathrm{n}=29)$ & $(\mathrm{n}=33)$ & $(\mathrm{n}=46)$ & $(\mathrm{n}=48)$ & $(\mathrm{n}=50)$ & $(\mathrm{n}=66)$ & $(\mathrm{n}=94)$ \\
\hline
\end{tabular}

"Autopsy bodies" refers to the total number of bodies imaged by CT scanning from January 2013 to September 2015 before autopsy. There were 782 males and 424 females (total, 1,206 bodies). The prior probability is the distribution of cases based on their ages (20s to 80 s) divided by the total number.

$\frac{P\left(I_{1} \mid A g e_{i}\right) \times P\left(I_{2} \mid A g e_{i}\right) \ldots \times P\left(I_{n} \mid A g e_{i}\right) \times P\left(A g e_{i}\right)}{\sum P\left(I_{1} \mid A g e_{j}\right) \times P\left(I_{2} \mid A g e_{j}\right) \ldots P\left(I_{n} \mid A g e_{j}\right) \times P\left(A g e_{j}\right)}$

After numerous observations are evaluated, all the probabilities for the distribution of age categories can be estimated from the observed data with Equation (3) for each individual (Lucy et al. 1996; Sakaue 2015).

In this Bayesian approach, the total data of the mean $\mathrm{HU}$ and the percentage of calcification for each case could be freely classified to create the estimation table. In this study, we sought to classify the data between 3 and 5 classes based on the mean HU and percentage of calcification using a scoring system. As the number of classes increased, the number of cases in the class decreased, and as the influence of variation, the hit rate is lowered. We first sorted the 136 study cases by sex and probable age in ascending order, and then classified the data into groups. We found that as the number of classes increased (i.e., 4 or 5 groups), the number of cases in each class decreased, and this affected both the degree of variation and the hit rate, with too few cases in some of the classes. Therefore, we found that the best compromise was to use three classes. The first class (score 1) included the lowest (1st) to the 23rd highest value cases from among the 70 male cases and the 1 st to 22 nd cases from among the 66 female cases. The second class (score 2) included the 24th to 46th highest value cases from among the remaining 47 male cases and the 23rd to 44th highest value cases from among the remaining 44 female cases. The third class (score 3 ) incorporated the remaining cases. The likelihood for each age group having all three classes was calculated for each sex. In addition, the presence or absence of calcification close to the sternal attachment region was calculated as a likelihood value for every age group (the presence of calcification has a score of 1 , and the absence of calcification has a score of 0 ). In summary, we created 12 scoring systems: 4 systems for the mean HU of the costal cartilage of both the second and fourth ribs, 4 systems for the percentage of calcification for the same cartilage regions, and 4 systems for the presence or absence of calcification in the same cartilage regions.

An age estimation table was created with each likelihood value, which could be used to estimate the posterior probability in each group. We examined the validity of this estimation table by comparing the actual age of 20 males and 20 females with the posterior probability of being placed in each age group.

\section{Results}

For our assessment of inter-observer error for the three observers, ICC values for the mean HU and percentage of calcification were 0.9962 and 0.9813 , respectively. Only one costal cartilage image resulted in different judgments among the observers: two observers judged that the calcification was present, and the other did that there was no calcification. For the other 39 costal cartilages, the observers were in complete agreement.

The Mann-Whitney $U$ test showed that mean HU and percentage of calcification were significantly higher $(P<$ 0.05 ) for females than those for males in the $60 \mathrm{~s}, 70 \mathrm{~s}$, and $80 \mathrm{~s}$ age groups in the left and right forth costal cartilages, except for $t$ the fourth costal cartilage in the 80s group on the left side. There were no significant sex differences with regards to mean $\mathrm{HU}$ or percentage of calcification for the left and right second costal cartilages among the age groups.

Overall, the mean HU and percentage of calcification seemed to increase with age for both sexes (Figs. 2 and 3). In males, however, there were significantly differences $(P<$ 0.05 ) only between cases in their 20s and 70s and cases in their $20 \mathrm{~s}$ and $80 \mathrm{~s}$ with regards to mean $\mathrm{HU}$, and between cases in their 20s and 70s with regards to percentage of calcification in both second costal cartilages. There were no significant differences among each age group with regards to mean HU and percentage of calcification for the left and right fourth costal cartilages. In females, we measured significant differences $(P<0.05)$ between cases in their $20 \mathrm{~s}$ and $60 \mathrm{~s}, 20 \mathrm{~s}$ and $70 \mathrm{~s}$, and $20 \mathrm{~s}$ and $80 \mathrm{~s}$ with regards to mean $\mathrm{HU}$ and percentage of calcification for the left second and both fourth costal cartilages. In addition, we found significant differences $(P<0.05)$ among female cases in their $20 \mathrm{~s}$ and $60 \mathrm{~s}$ and in their $20 \mathrm{~s}$ and $80 \mathrm{~s}$ with regard to the mean HU for the right second costal cartilage.

Despite these trends, there was marked variation across individuals within many age groups (Figs. 4, 5, 6 and 7). Fig. 8 shows a scatter plot of all 136 study cases for the mean HU and percentage of calcification. The coefficient of determination $\left(R^{2}\right)$ was greater than 0.8 in all four regions, and we determined a positive correlation between 

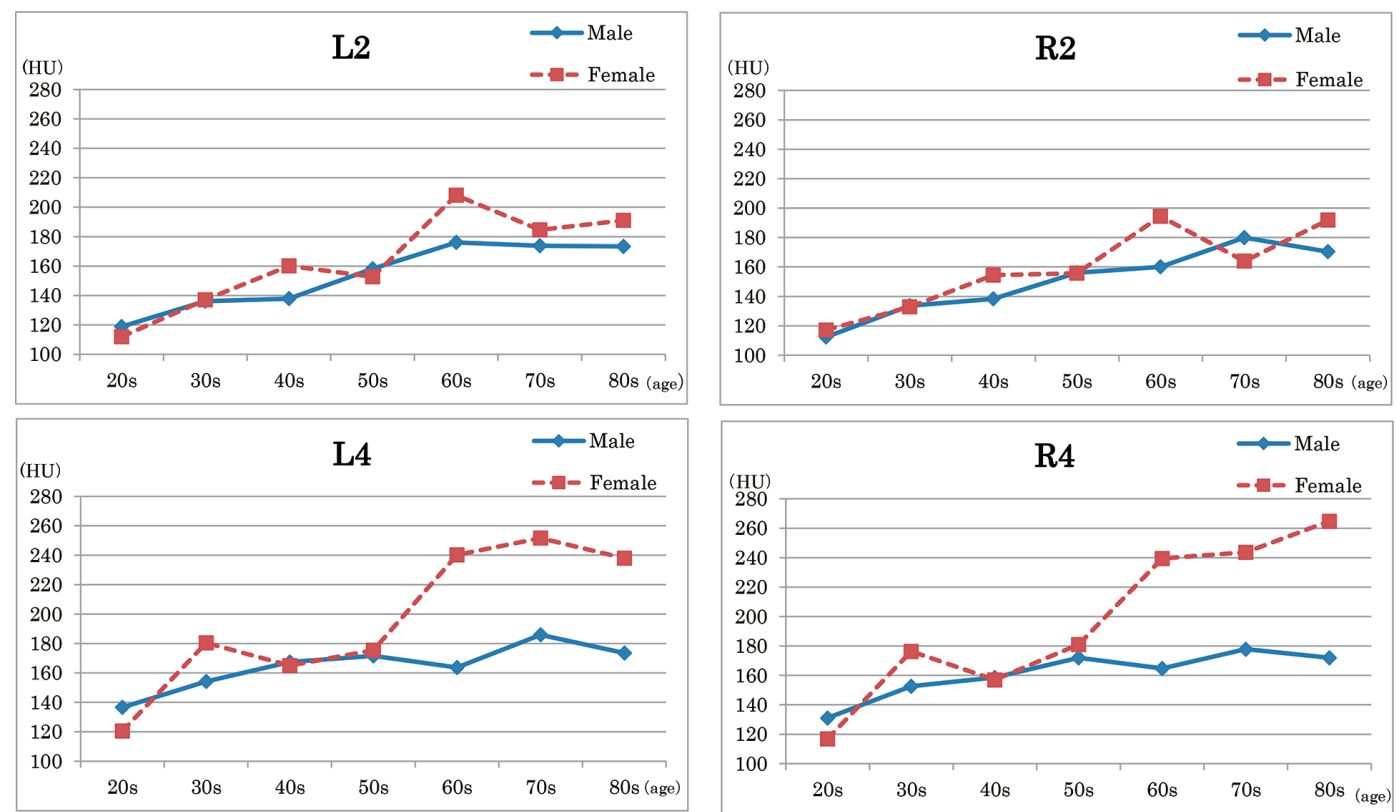

Fig. 2. Relationship between age group and mean Hounsfield unit (HU)

The mean HU was significantly higher for females $(P<0.05)$ than males in the 60 - to 80 -year age ranges for the left and right second and fourth costal cartilages, except for the 80 s group and the left fourth costal cartilage. For males, there were significant differences $(P<0.05)$ between cases in their $20 \mathrm{~s}$ and $70 \mathrm{~s}$ and cases in their $20 \mathrm{~s}$ and $80 \mathrm{~s}$ for both second costal cartilages. In females, there were significant differences $(P<0.05)$ between cases in their $20 \mathrm{~s}$ and $60 \mathrm{~s}, 20 \mathrm{~s}$ and $70 \mathrm{~s}$, and $20 \mathrm{~s}$ and $80 \mathrm{~s}$ for the left second and both fourth costal cartilages, and between cases in their 20s and $60 \mathrm{~s}$ and cases in their $20 \mathrm{~s}$ and $80 \mathrm{~s}$ for the right second costal cartilage.

L: left, R: right, 2: 2nd cartilage, 4; 4th cartilage.
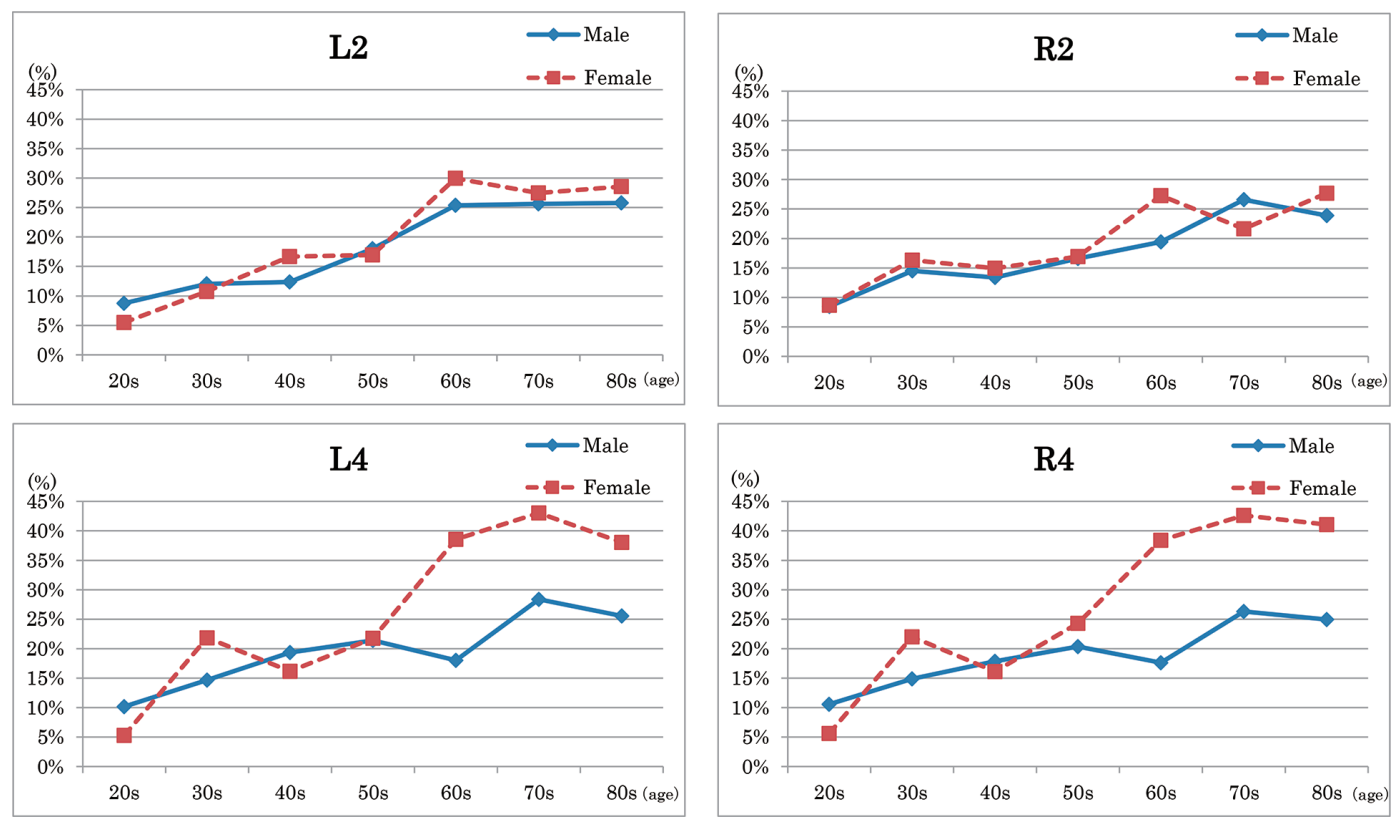

Fig. 3. Relationship between age and percentage of calcification.

The percentage of calcification was significantly higher for females $(P<0.05)$ than for males, as noted for the mean HU. In males, there were significant differences $(P<0.05)$ between cases in their $20 \mathrm{~s}$ and $70 \mathrm{~s}$ with regards to percentage of calcification in both second costal cartilages. In females, there were significant differences $(P<0.05)$ between cases in their $20 \mathrm{~s}$ and $60 \mathrm{~s}, 20 \mathrm{~s}$ and $70 \mathrm{~s}$, and $20 \mathrm{~s}$ and $80 \mathrm{~s}$ with regards to percentage of calcification in the left second and both fourth costal cartilages.

L, left; R, right; 2, 2nd cartilage; 4, 4th cartilage. 

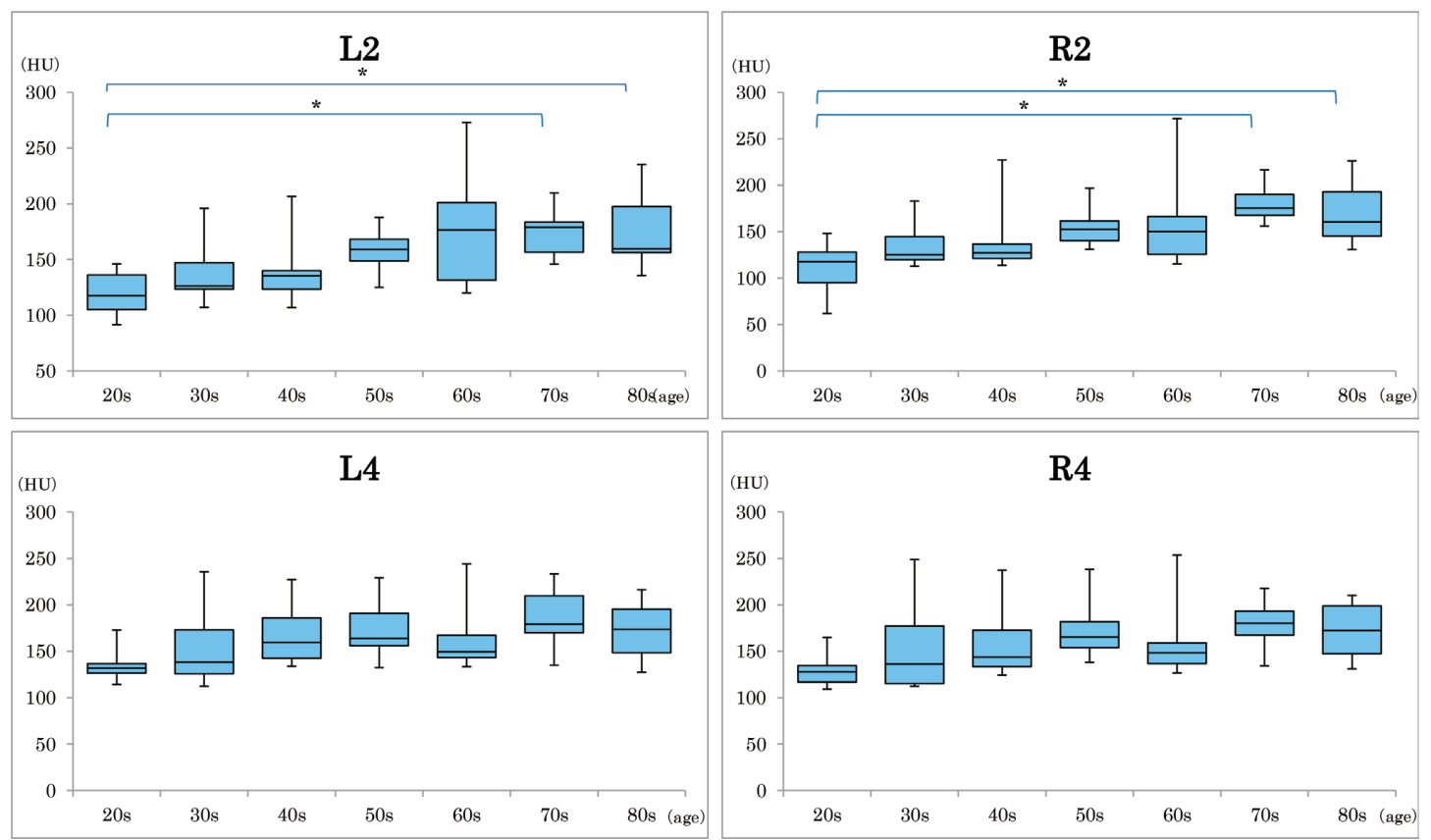

Fig. 4. Box-and-whisker plot of mean Hounsfield unit (HU) for each age group in males. $\mathrm{L}$, left; R, right; 2, 2nd cartilage; 4, 4th cartilage. $* P<0.05$.
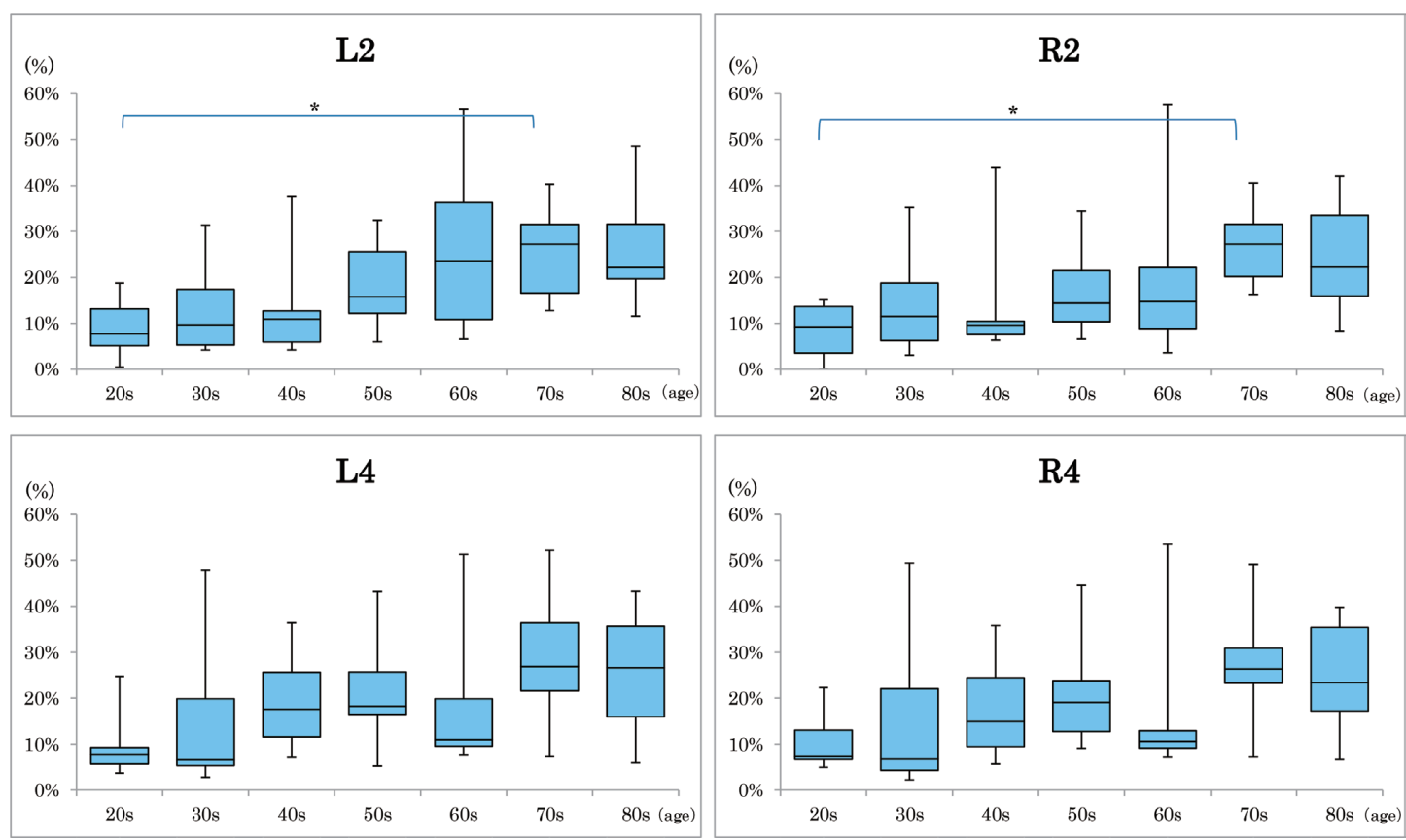

Fig. 5. Box-and-whisker plot of the percentage of calcification for each age group in males.

L, left; R, right; 2, 2nd cartilage; 4, 4th cartilage. ${ }^{*} P<0.05$.

the mean HU and the percentage of calcification.

Based on our Bayesian calculations, only 15 out of 40 sample cases had the highest probability of correctly estimating age group, including eight of 20 males $(40 \%)$ and seven of 20 females $(35 \%)$ (Table 2). The hit rate when using three consecutive age groups (i.e., the highest probability age group plus one age group on either side) reached $65 \%$ for male cases $(n=13)$ and $75 \%$ for female cases $(n=$
15) (Table 2). However, in five cases, the actual age was not included in any of the estimated age groups. These were three males aged 42,58 , and 88 years, and two females aged 65 and 75 years in whom a widespread area of costal cartilage was not ossified.

\section{Discussion}

In regard to the mean $\mathrm{HU}$ and percentage of calcifica- 

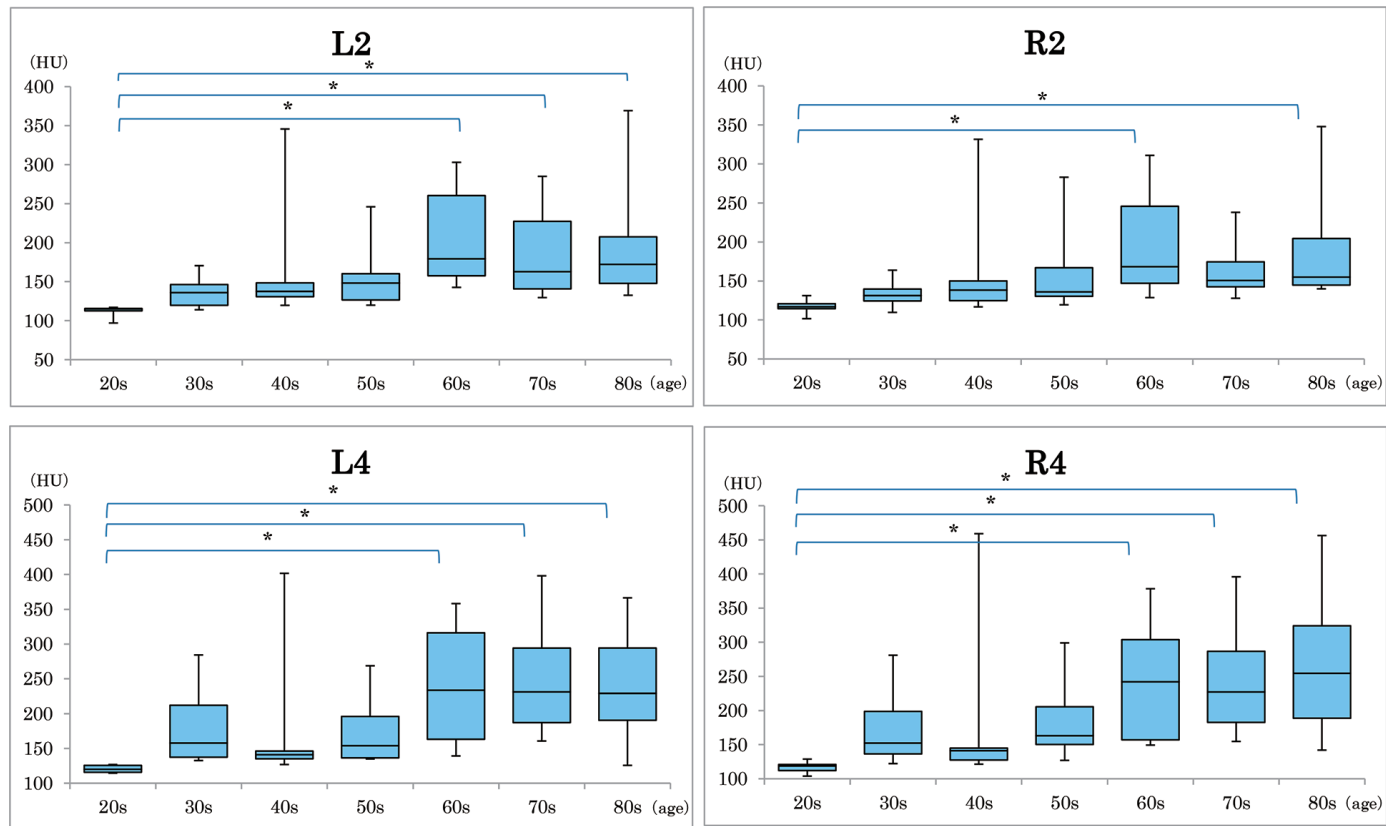

Fig. 6. Box-and-whisker plot of Hounsfield unit (HU) for each age group in females.

$\mathrm{L}$, left; R, right; 2, 2nd cartilage; 4, 4th cartilage. ${ }^{*} P<0.05$.
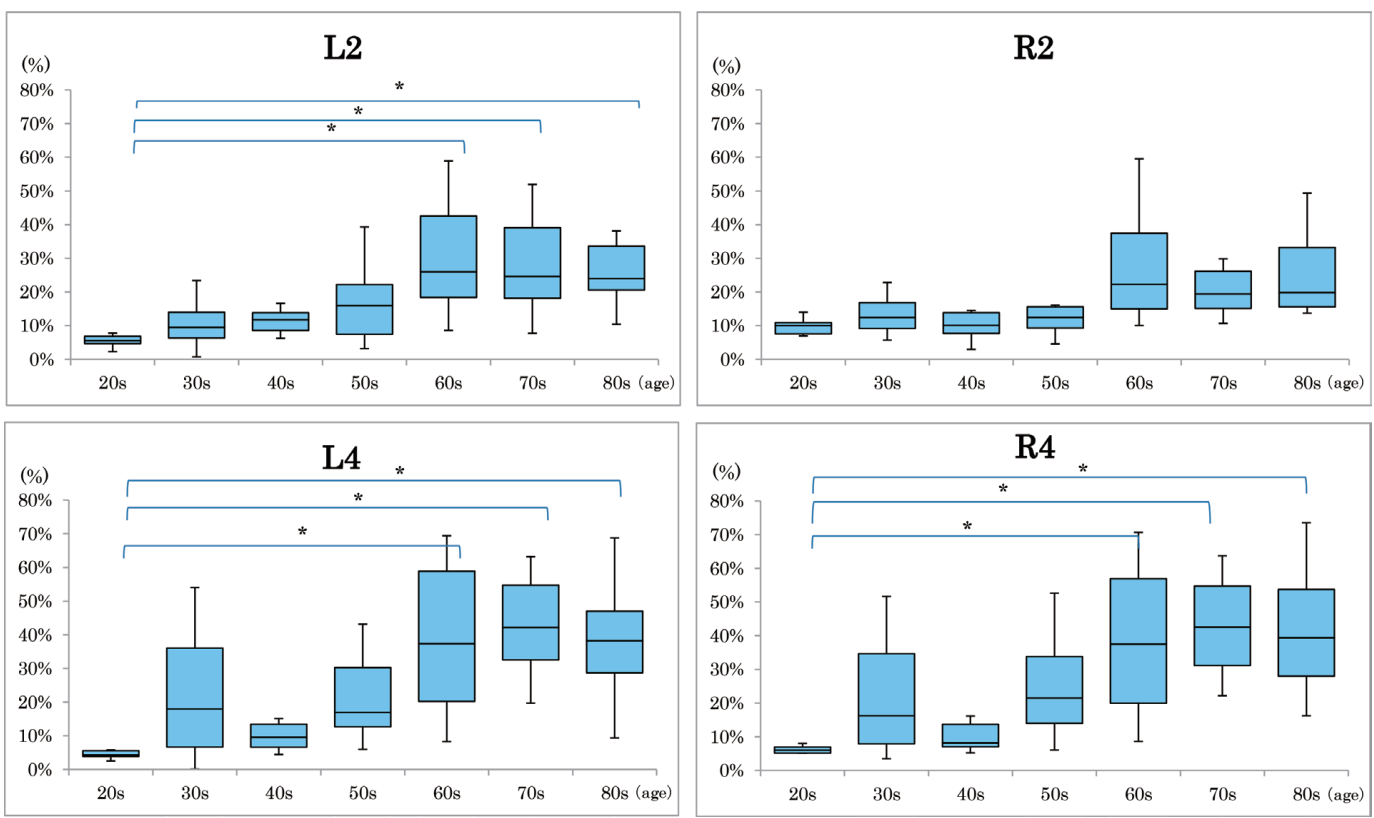

Fig. 7. Box-and-whisker plot of the percentage of calcification in each age group for females.

L, left; R, right; 2, 2nd cartilage; 4, 4th cartilage. ${ }^{*} P<0.05$.

tion, we observed marked individual differences in many of the age groups, and this variability makes it difficult to estimate age. Therefore, we considered the utility of Bayesian statistics to create a reference age estimation table. Bayesian statistics can be applied effectively to a population that contains highly variable individual data. When using a Bayesian method, establishing the prior probability is essential. Recently, Aramaki et al. (2017) challenged the mode of analysis of thyroid cartilage ossification using Bayesian estimation and $\mathrm{CT}$ imaging to estimate age at death. The present study echoes the viewpoint raised by Aramaki and colleagues that Bayesian estimation could be a useful tool for age determination studies. Given that different parts of the body are often found at crime scenes, it is important to confirm that other regions can be similarly used for age estimation. In this study, I used the age distribution of consecutive forensic autopsy cases from our institution for which there were available postmortem CT data to estimate the prior probability. Konigsberg et al. (2008) said that if appropriate prior age-at-death distributions are 

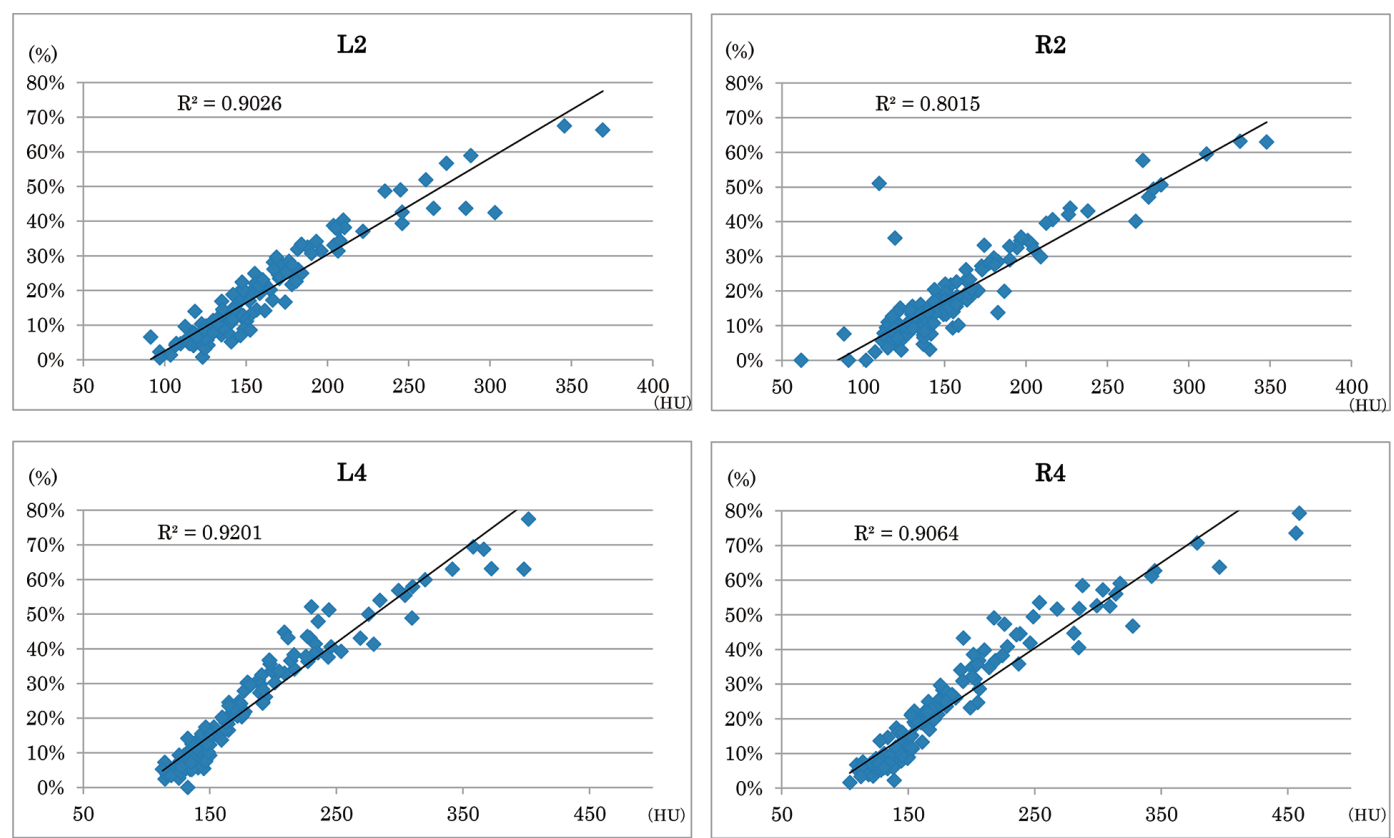

Fig. 8. Scatter plot of the percentage of calcification and mean Hounsfield unit (HU).

Scatter plot of all 136 study cases with the percentage of calcification on the $y$-axis and mean HU on the x-axis. The coefficient of determination $\left(R^{2}\right)$ was greater than 0.8 in all four regions, and there was a strong correlation between mean HU and the percentage of calcification.

L, left; R, right; 2, 2nd cartilage; 4, 4th cartilage.

Table 2. Posterior probability table based on age estimation.

\begin{tabular}{|c|c|c|c|c|c|c|c|c|c|c|c|c|c|c|c|c|c|}
\hline \multirow{2}{*}{$\begin{array}{c}\text { Age } \\
\text { (years) }\end{array}$} & \multirow{2}{*}{ Sex } & \multicolumn{7}{|c|}{ Posterior probability (\%) } & \multirow{2}{*}{$\begin{array}{c}\text { Age } \\
\text { (years) }\end{array}$} & \multirow{2}{*}{ Sex } & \multicolumn{7}{|c|}{ Posterior probability (\%) } \\
\hline & & $20 \mathrm{~s}$ & $30 \mathrm{~s}$ & $40 \mathrm{~s}$ & $50 \mathrm{~s}$ & $60 \mathrm{~s}$ & $70 \mathrm{~s}$ & $80 \mathrm{~s}$ & & & $20 \mathrm{~s}$ & $30 \mathrm{~s}$ & $40 \mathrm{~s}$ & $50 \mathrm{~s}$ & $60 \mathrm{~s}$ & $70 \mathrm{~s}$ & $80 \mathrm{~s}$ \\
\hline 25 & $\mathrm{M}$ & 74 & 26 & 1 & 0 & $<0.5$ & 0 & 0 & 28 & $\mathrm{~F}$ & 98 & 1 & 1 & 0 & 0 & 0 & 0 \\
\hline 34 & M & 24 & 66 & 6 & 0 & 4 & 0 & 0 & 30 & $\mathrm{~F}$ & 99 & $<0.5$ & $<0.5$ & 0 & 0 & 0 & 0 \\
\hline 36 & M & 2 & 14 & 62 & 14 & 7 & 0 & 1 & 33 & $\mathrm{~F}$ & 58 & $<0.5$ & 40 & 1 & 0 & 0 & 0 \\
\hline 42 & $\mathrm{M}$ & 97 & 3 & 0 & 0 & 0 & 0 & 0 & 38 & $\mathrm{~F}$ & 0 & $<0.5$ & $<0.5$ & 20 & 4 & 24 & 51 \\
\hline 42 & $\mathrm{M}$ & 24 & 66 & 6 & 0 & 4 & 0 & 0 & 42 & $\mathrm{~F}$ & 0 & $<0.5$ & 95 & 4 & $<0.5$ & 0 & 0 \\
\hline 42 & M & 1 & 1 & 9 & 79 & 7 & 1 & 4 & 48 & $\mathrm{~F}$ & 26 & $<0.5$ & 72 & 2 & 0 & 0 & 0 \\
\hline 51 & $\mathrm{M}$ & 0 & $<0.5$ & 1 & 86 & 1 & 2 & 10 & 49 & $\mathrm{~F}$ & 0 & $<0.5$ & 98 & 2 & $<0.5$ & 0 & 0 \\
\hline 53 & M & 0 & $<0.5$ & $<0.5$ & 1 & $<0.5$ & 91 & 8 & 50 & $\mathrm{~F}$ & 0 & $<0.5$ & 81 & 17 & 0 & 0 & 1 \\
\hline 53 & $\mathrm{M}$ & 1 & 1 & 5 & 83 & 4 & 1 & 5 & 57 & $\mathrm{~F}$ & 0 & $<0.5$ & $<0.5$ & $<0.5$ & 15 & 21 & 63 \\
\hline 58 & $\mathrm{M}$ & 24 & 66 & 6 & 0 & 4 & 0 & 0 & 57 & $\mathrm{~F}$ & 0 & 2 & 1 & 10 & 58 & 30 & 0 \\
\hline 60 & $\mathrm{M}$ & 74 & 26 & 1 & 0 & $<0.5$ & 0 & 0 & 63 & $\mathrm{~F}$ & 0 & 0 & 49 & 47 & 4 & 0 & 0 \\
\hline 61 & M & 0 & $<0.5$ & $<0.5$ & 9 & 58 & 25 & 8 & 65 & $\mathrm{~F}$ & 0 & 2 & 74 & 24 & 0 & 0 & 0 \\
\hline 64 & $\mathrm{M}$ & 3 & 33 & 54 & 0 & 10 & 0 & 0 & 66 & $\mathrm{~F}$ & 0 & $<0.5$ & $<0.5$ & 20 & 4 & 24 & 51 \\
\hline 69 & $\mathrm{M}$ & 0 & $<0.5$ & $<0.5$ & 1 & $<0.5$ & 91 & 8 & 69 & $\mathrm{~F}$ & 0 & $<0.5$ & $<0.5$ & 1 & 3 & 25 & 71 \\
\hline 71 & M & 0 & $<0.5$ & $<0.5$ & 20 & 27 & 30 & 22 & 75 & $\mathrm{~F}$ & 0 & 5 & 19 & 76 & 0 & 0 & 0 \\
\hline 72 & $\mathrm{M}$ & 0 & $<0.7$ & $<0.5$ & 11 & 3 & 62 & 24 & 75 & $\mathrm{~F}$ & 0 & 0 & $<0.5$ & $<0.5$ & 34 & 26 & 39 \\
\hline 75 & $\mathrm{M}$ & 0 & $<0.5$ & $<0.5$ & 1 & $<0.5$ & 91 & 8 & 78 & $\mathrm{~F}$ & 0 & $<0.5$ & $<0.5$ & $<0.5$ & 0 & 31 & 69 \\
\hline 82 & $\mathrm{M}$ & $<0.5$ & $<0.5$ & 1 & 90 & 1 & 1 & 7 & 80 & $\mathrm{~F}$ & 0 & 0 & $<0.5$ & $<0.5$ & 32 & 18 & 50 \\
\hline 84 & $\mathrm{M}$ & 0 & $<0.5$ & $<0.5$ & 1 & $<0.5$ & 91 & 8 & 86 & $\mathrm{~F}$ & 0 & $<0.5$ & $<0.5$ & 20 & 4 & 24 & 51 \\
\hline 88 & $\mathrm{M}$ & $<0.5$ & 2 & 66 & 0 & 32 & 0 & 0 & 88 & $\mathrm{~F}$ & 0 & $<0.5$ & $<0.5$ & $<0.5$ & 19 & 14 & 67 \\
\hline
\end{tabular}

Gray cells show three consecutive age groups that include the highest probability age group plus one age group on either side. When the highest probability age group is the $20 \mathrm{~s}$ or $80 \mathrm{~s}$ groups, the three consecutive age groups are $20 \mathrm{~s}, 30 \mathrm{~s}, 40 \mathrm{~s}$, and $60 \mathrm{~s}, 70 \mathrm{~s}, 80 \mathrm{~s}$, respectively. When a posterior probability of 0 is included in any of the three age groups, that age group was deleted and only two groups are shown. If the actual age was included in the gray cells, it was assumed that age estimation was carried out correctly.

M, male; F, female. 
used, the analysis will provide an accurate estimation.

Here, we sought to apply our findings to estimate age in forensic cases, except for cases with severely decomposed bodies. In our institutions, severely decomposed bodies do not undergo CT scanning. In addition, this study primarily focused on confirming whether Bayesian estimation could be useful for estimating age using CT imaging. However, the field of forensics often requires age estimation to be carried out on bodies at various stage of decomposition. Therefore, for its broader applicability in the field, it will be important to extend upon the current work using costal cartilages at various stages of decomposition to estimate age.

In the present study, to verify prior probability, we measured the age composition of a male population in Japan of 2016, and we found little difference in the results. We selected 20 males and 20 females from our autopsy cases (one to four individuals per age group) and entered these cases into the age estimation table (consisting of the 136 study cases). Among these 40 cases, we were unable to correctly classify the actual age for three males and two females (Table 2). However, we noticed that calcification was lower than would be expected for the age in each of these five cases. This scenario could easily occur when the reference population does not include samples that greatly deviate from the mean. As stated above, costal cartilage calcification can differ markedly across individuals, so this outcome is not surprising. The advantage of Bayesian estimation is that one can sequentially update the reference estimation table by adding more data, as they are collected. Using this updated table, the age of the next case could be estimated more accurately.

In Bayesian statistics, probability forms a distribution for individuals who have similar characteristics. The age group with the highest probability does not directly correspond to the most probable age group. In practice, however, we have shown that it would be reasonable to consider the age group with the highest probability as the actual age group. In 40 samples (20 males and 20 females), we had matches for highest probability age and actual age for only eight males (40\%) and seven females (35\%) (Table 2). We hypothesize that the proportion of correct matching might be low because of the 10-year-unit cut offs. For example, a 39 -year-old or 50-year-old individual may have characteristics of the 40-year age group, rather than their actual age groups of $30 \mathrm{~s}$ or $50 \mathrm{~s}$. Thus, if the range of age groups is extended, we would expect an increase in the percentage of correctly matched cases. Indeed, when we considered three consecutive age groups, the number of correctly matched cases reached 13 for males (65\%) and 15 for females (75\%) (Table 2). Iscan et al. (1984a) claimed that costal cartilages can be used for estimating ages under 30 years (range of 3 to 5 years) and over 30 years old (range of 8 to 13 years) in males, and under 31 years (range, 4 to 6 years) and over 33 years (range, 11 to 15 years) in females (Iscan et al. 1985). However, the authors note that the estimation error is large for cases over 40 years (Cerezo-Roman and Hernandez Espinoza 2014; Haj Salem et al. 2014) and this is also associated with a larger inter-observer error. There are few methods to estimate age in older people because of large individual differences (Kimmerle et al. 2008; Fanton et al. 2010; Hartnett 2010). Our method offers a solution to this, and allows us to better estimate age without bias in all ages with low inter-observer error.

Our image processing procedure is a limitation of this study. The mean HU is the average tissue absorbance by volume, which can vary depending on the size and characteristics of the subject and the CT parameters. The age estimation table we created for this study may not be directly applicable to CT images taken at other institutions. Thus, each institute might need to create its own estimation table.

In general, when performing age estimations, it is more common to include a broader age range to reduce the risk of exclusion. Individual differences in costal cartilage calcification can be large, and it is difficult to use traditional methods of age estimation without introducing observer error. Based on our results, it seems that our method was effectively free from inter-observer error. We anticipate that Bayesian statistics could be used in conjunction with other techniques in the field to help ascertain age at death, particularly in cases that prove difficult to solve with conventional methods alone.

\section{Acknowledgments}

The author wishs to acknowledge Dr. K. Sakaue, National Museum of Nature and Science, for his valuable advice and constructive discussion and Dr. A. Usui, Departments of Diagnostic Image Analysis Tohoku University Graduate School of Medicine, for technical assistance with the experiments. I also grateful to Dr. M. Funayama, Professor of Departments of Forensic Medicine Tohoku University Graduate School of Medicine, for his help in interpreting the significance of the results of this study.

\section{Conflict of Interest}

The author declares no conflict of interest.

\section{References}

Aramaki, T., Ikeda, T., Usui, A. \& Funayama, M. (2017) Age estimation by ossification of thyroid cartilage of Japanese males using Bayesian analysis of postmortem CT images. Leg. Med. (Tokyo), 25, 29-35.

Barchilon, V., Hershkovitz, I., Rothschild, B.M., Wish-Baratz, S., Latimer, B., Jellema, L.M., Hallel, T. \& Arensburg, B. (1996) Factors affecting the rate and pattern of the first costal cartilage ossification. Am. J. Forensic Med. Pathol., 17, 239-247.

Cerezo-Roman, J.I. \& Hernandez Espinoza, P.O. (2014) Estimating age at death using the sternal end of the fourth ribs from Mexican males. Forensic Sci. Int., 236, 196 e1-6.

Dedouit, F., Bindel, S., Gainza, D., Blanc, A., Joffre, F., Rouge, D. \& Telmon, N. (2008) Application of the Iscan method to twoand three-dimensional imaging of the sternal end of the right fourth rib. J. Forensic Sci., 53, 288-295.

Fanton, L., Gustin, M.P., Paultre, U., Schrag, B. \& Malicier, D. (2010) Critical study of observation of the sternal end of the right 4th rib. J. Forensic Sci., 55, 467-472. 
Garamendi, P.M., Landa, M.I., Botella, M.C. \& Aleman, I. (2011) Forensic age estimation on digital $\mathrm{X}$-ray images: medial epiphyses of the clavicle and first rib ossification in relation to chronological age. J. Forensic Sci., 56 Suppl 1, S3-12.

Haj Salem, N., Aissaoui, A., Mesrati, M.A., Belhadj, M., Quatrehomme, G. \& Chadly, A. (2014) Age estimation from the sternal end of the fourth rib: a study of the validity of Iscan's Method in Tunisian male population. Leg. Med. (Tokyo), 16, 385-389.

Hartnett, K.M. (2010) Analysis of age-at-death estimation using data from a new, modern autopsy sample-part II: sternal end of the fourth rib. J. Forensic Sci., 55, 1152-1156.

Inoi, T. (1997) Estimation of sex and age by calcification pattern of costal cartilage in Japanese. Nihon Hoigaku Zasshi, 51, 89-94.

Iscan, M.Y., Loth, S.R. \& Wright, R.K. (1984a) Age estimation from the rib by phase analysis: white males. J. Forensic Sci., 29, 1094-1104.

Iscan, M.Y., Loth, S.R. \& Wright, R.K. (1984b) Metamorphosis at the sternal rib end: a new method to estimate age at death in white males. Am. J. Phys. Anthropol., 65, 147-156.

Iscan, M.Y., Loth, S.R. \& Wright, R.K. (1985) Age estimation from the rib by phase analysis: white females. J. Forensic Sci., 30, 853-863.

Kampen, W.U., Claassen, H. \& Kirsch, T. (1995) Mineralization and osteogenesis in the human first rib cartilage. Ann. Anat., 177, 171-177.

King, J.B. (1939) Calcification of the costal cartilages. Br. J. Radiol., 12, 2-12.

Kimmerle, E.H., Prince, D.A. \& Berg, G.E. (2008) Inter-observer variation in methodologies involving the pubic symphysis, sternal ribs, and teeth. J. Forensic Sci., 53, 594-600.
Konigsberg, L.W., Herrmann, N.P., Wescott, D.J. \& Kimmerle, E.H. (2008) Estimation and evidence in forensic anthropology: age-at-death. J. Forensic Sci., 53, 541-557.

Kunos, C.A., Simpson, S.W., Russell, K.F. \& Hershkovitz, I. (1999) First rib metamorphosis: its possible utility for human age-at-death estimation. Am. J. Phys. Anthropol., 110, 303-323.

Lucy, D., Aykroyd, R.G., Pollard, A.M. \& Solheim, T. (1996) A Bayesian approach to adult human age estimation from dental observations by Johanson's age changes. J. Forensic Sci., 41, 189-194.

McCormick, W.F. (1980) Mineralization of the costal cartilages as an indicator of age: preliminary observations. J. Forensic Sci., 25, 736-741.

Rejtarova, O., Hejna, P., Soukup, T. \& Kuchar, M. (2009) Age and sexually dimorphic changes in costal cartilages. A preliminary microscopic study. Forensic Sci. Int., 193, 72-78.

Rejtarova, O., Slizova, D., Smoranc, P., Rejtar, P. \& Bukac, J. (2004) Costal cartilages: a clue for determination of sex. Biomed. Pap. Med. Fac. Univ. Palacky Olomouc Czech. Repub., 148, 241-243.

Sakaue, K. (2015) A Bayesian approach to age estimation from cranial suture closure in Japanese people. Bull. Natl. Mus. Nat. Sci., Ser. D, 41, 1-11.

Stewart, J.H. \& McCormick, W.F. (1984) A sex- and age-limited ossification pattern in human costal cartilages. Am. J. Clin. Pathol., 81, 765-769.

Verzeletti, A., Terlisio, M. \& De Ferrari, F. (2013) Age-at-death estimation in Caucasian females from the morphological analysis of the sternal end of the fourth rib. Leg. Med. (Tokyo), 15, 47-49. 